\title{
Coverillustration
}

\section{Hiding in plain view}

C mouflage is essential for any prey and evolution has provided that mysterious sleight of hand ability to many successful herbivorous species. Some of these tricks would be the envy of the best of magicians. Yet, the camouflage cannot be so good that communication is stilled within the species, limiting social interactions such as courting and mating. Consider the bullethead parrotfish (Chlorurus sordidus) on this month's cover as it uses the technique of pointillism to hide in plain view at a predatorial distance and yet keeps its harem in order at a closer distance.

Pointillism is a technique in painting whereby small dots of "pure" colours are used to create an overall effect of "blended" colours when viewed from the proper distance. Theoretically, the technique would increase luminosity to achieve an optical blending at the retinal level. This artistic idea was demonstrated by Georges Seurat in 1884 in his painting "A Sunday Afternoon on the Island of the Grand Jatte." He was not, however, the first nor the last to utilise this principle. The technique relies upon the concept of receptive fields although the neo-impressionists were unaware of this aspect of visual pathway organisation. At a certain distance a "pure" coloured dot will stimulate a single cone or small group of cones, contributing to a centre surround receptive field. The brightly coloured dot will be seen in its original colour, unless the painting is viewed from a more distant point. With increasing distance, the adjacent dots will stimulate the same receptive field with two or more colours. If those colours are complementary, the resulting neural signal will be an achromatic dull intermediate colour. That is the difficulty with the technique and the reason the artistic principle of pointillism was eventually abandoned by many artists. Not by the parrotfish, however, as becoming duller at distance is exactly what they need for survival.

Most male parrotfish and their kin perform a disappearing act by having two adjacent complementary colours on their bodies. When viewed at close range, the males appear bright and colourful, and this is important for communication

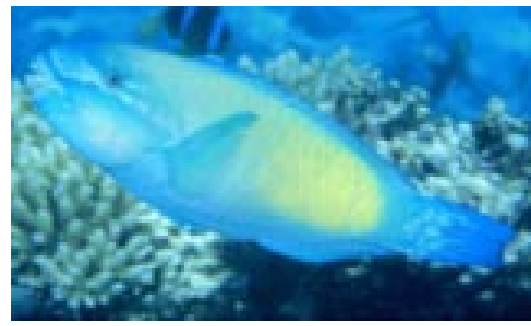

antimicrobial peptides resembling the defence peptides discussed in the BJO cover essay of July 2000.

The parrotfish family (Scaridae) is evolutionarily quite young, probably originating approximately 12 million years ago in the Tethys Sea, a geological precursor of the Indo-Pacific tropical waters. The transformation into the highly colourful and numerically dominant coral reef fish has been rapid with a profound selection pressure directed towards male coloration.

Although the eyes of this particular species have not been described in detail, some general trends in reef fish vision are as follows. Rods are not found in the shallow water larval forms but are initially seen in the juveniles and increase in the adult forms as the fish settle to deeper waters. As the eye matures, additional photoreceptors are added and the cones in particular become more organised to improve acuity. The adult fish has a retinal mosaic with single and double cones conferring a visual potential of approximately 10 arc minutes, which is a considerable improvement over the larval and juvenile forms. Although these fish have a predominantly cone retina, there are some rods for lower light situations. Although the sensitivity of the visual photopigments has not been measured, there are suggestions that ultraviolet and polarised light may be recognised, at least in the juvenile forms.

Finally, the last disappearing act to mention is a sexual one that defies imagination. If a harem loses its male, the dominant female of the harem will transform into a male including the development of the bright coloration. To further mystify, this male can return to being a drab female if conditions require it. It is amazing how this species can hide in plain view.

crete their own pyjamas. A mucous gland in the oropharynx at the base of the gills secretes a mucous sac that surrounds the fish during darkness allowing the fish to "sleep." If the mucous sac is disturbed the fish will rapidly exit using the mucous envelope as a protective mechanism. This mucous sac protects against predators and parasites as well as limiting the olfactory stimuli for nocturnal hunters such as the moray eel. For added protection, the mucous sac is filled with 